\title{
Effect of oral eicosapentaenoic acid on epidermal Langerhans cell numbers and PGD2 production in UVR- exposed human skin: a randomised controlled study
} DOI:

10.1111/exd.13177

\section{Document Version \\ Accepted author manuscript}

Link to publication record in Manchester Research Explorer

Citation for published version (APA):

Pilkington, S., Gibbs, N., Costello, P., Bennett, S., Massey, K. A., Friedmann, P. S., Nicolaou, A., \& Rhodes, L. (2016). Effect of oral eicosapentaenoic acid on epidermal Langerhans cell numbers and PGD2 production in UVRexposed human skin: a randomised controlled study. Experimental Dermatology. https://doi.org/10.1111/exd.13177

\section{Published in:}

Experimental Dermatology

\section{Citing this paper}

Please note that where the full-text provided on Manchester Research Explorer is the Author Accepted Manuscript or Proof version this may differ from the final Published version. If citing, it is advised that you check and use the publisher's definitive version.

\section{General rights}

Copyright and moral rights for the publications made accessible in the Research Explorer are retained by the authors and/or other copyright owners and it is a condition of accessing publications that users recognise and abide by the legal requirements associated with these rights.

\section{Takedown policy}

If you believe that this document breaches copyright please refer to the University of Manchester's Takedown Procedures [http://man.ac.uk/04Y6Bo] or contact uml.scholarlycommunications@manchester.ac.uk providing relevant details, so we can investigate your claim.

\section{OPEN ACCESS}


1 Effect of oral eicosapentaenoic acid on epidermal Langerhans cell numbers and $\mathrm{PGD}_{2}$

2 production in UVR-exposed human skin: a randomised controlled study

3

4 Suzanne M Pilkington ${ }^{1}$, Neil K Gibbs ${ }^{1}$, Patrick Costello', Susan P Bennett ${ }^{3}$, Karen A

5 Massey $^{2}$, Peter S Friedmann ${ }^{4}$, Anna Nicolaou ${ }^{2}$, Lesley E Rhodes ${ }^{1,3}$

$6 \quad{ }^{1}$ Centre for Dermatology, Institute of Inflammation and Repair and ${ }^{2}$ School of Pharmacy,

7 Faculty of Medical and Human Sciences, University of Manchester, Manchester, UK;

$8 \quad{ }^{3}$ Centre for Dermatology, Salford Royal Hospital, Manchester Academic Health Science

9 Centre, Manchester, UK; and ${ }^{4}$ Faculty of Medicine, University of Southampton,

10 Southampton, UK.

Correspondence: Prof Lesley Rhodes, Photobiology Unit, Centre for Dermatology, University of Manchester, Salford Royal Hospital, Manchester, UK.

Tel 0044161206 1150; Email lesley.e.rhodes@manchester.ac.uk

Keywords: Photoimmunosuppression, dendritic cells, prostaglandin $D_{2}$, omega-3 fatty acids; systemic photoprotection 


\section{Abstract}

Langerhans cells (LC) are sentinels of skin's immune system, their loss from epidermis contributing to UVR-suppression of cell mediated immunity (CMI). Omega-3 polyunsaturated fatty acids ean show potential to abrogate UVR-suppression of CMI in mice and humans, potentially through modulation of LC migration. Our objectives were to examine if eicosapentaenoic acid (EPA) ingestion influences UV-mediated effects on epidermal LC numbers and levels of immunomodulatory mediators including prostaglandin $(P G) D_{2}$, which is expressed by LC.

In a double-blind randomised controlled study, healthy individuals took $5 \mathrm{~g}$ EPA-rich $(n=40)$ or control $(n=33)$ lipid for 12-weeks; UVR exposed and unexposed skin samples were taken pre- and post-supplementation. Epidermal LC numbers were assessed by immunofluorescence for CD1a, and skin blister fluid PG and cytokines quantified by LCMS/MS and Luminex assay, respectively. Pre-supplementation, UVR reduced mean (SEM) LC number/mm² from $913(28)$ to $322(40)(p<0.001)$, and mean $P_{G D_{2}}$ level by $37 \%$ from 8.1 (11.6) to 5.1 (5.6) $\mathrm{pg} / \mu \mathrm{l} ; p<0.001)$, while IL-8 level increased $(p<0.001)$. Despite confirmation of EPA bioavailability in red blood cells and skin in the active group, no between-group effect of EPA was found on UVR-modulation of LC numbers, $\mathrm{PGD}_{2}$ or cytokine levels postsupplementation.

Thus no evidence was found for EPA abrogation of photoimmunosuppression through an impact on epidermal LC numbers. Intriguingly, UVR-exposure substantially reduced cutaneous $\mathrm{PGD}_{2}$ levels in humans, starkly contrasting with reported effects of UVR on other skin PG. Lowered $P G D_{2}$ levels could reflect LC loss from the epidermis and/or altered dendritic cell activity, and may be relevant for phototherapy of skin disease. 


\section{Introduction}

Ultraviolet radiation (UVR) suppresses cutaneous immunity (photoimmunosuppression) and this is believed to be an important contributor to the development of skin cancers [1]. In addition to the mutagenic effects of UVR on DNA which initiate carcinogenesis, inhibition of cell mediated immunity (CMI) can allow cancerous cells to escape destruction by cytotoxic lymphocytes, facilitating tumour progression. This has been elegantly demonstrated in mouse models where antigenic tumour cells were transplanted into UVR-exposed mice where they were able to progress [2]. Moreover, immunosuppressed patients have a higher incidence of skin malignancies [3].

Dendritic cells, including epidermal Langerhans cells (LC) and dermal dendritic cells (DC), are antigen presenting cells (APC) and are amongst the first line of defence in the skin where they facilitate innate and adaptive immunity and promote antigenic tolerance $[4,5]$. The LC reside above the basal layer of the epidermis and monitor the skin microenvironment for danger signals including pathogens, chemicals and tumour peptides. On capturing antigenic material they travel along the afferent lymphatics to the skin-draining lymph nodes (DLN) and activate differentiation of naïve T cells (Th-0) into T helper (Th)-1, Th-2, Th17, Th22 or Treg cells [5]. Following UVR-exposure LC migrate away from the epidermis [6], and their behaviour is altered, favouring activation of Th-2 immune responses and Treg cells over Th-1 driven CMI [7-10]; these changes are believed to contribute to UVR-induced suppression of skin immunity [11]. This can be observed clinically by diminished skin contact hypersensitivity (CHS) and delayed type hypersensitivity responses to allergens following UVR-exposure [12].

The response of LC and other dendritic cells to antigen are strongly influenced by signals in the skin microenvironment. Cytokines TNF- $\alpha$ and IL-1 $\beta$ stimulate LC migration from the epidermis after exposure to antigen $[13,14]$, and both are upregulated in the skin in response to UVR-exposure. UVR also upregulates further cytokines possessing proinflammatory (including IL-8, IL-6 and IFN- $\gamma$ ) and immunosuppressive (including IL-4 and IL- 
10) properties $[15,16]$. Moreover, prostaglandins (PG) produced in the skin are reported to regulate dendritic cell activity. $\mathrm{PGE}_{2}$ can modulate LC migration and maturation in mice [17] and reduces the ability of bone marrow derived dendritic cells to stimulate a CMI responses following UVR-exposure [18], indicating a potential influence on antigen presenting activity during photoimmunosuppression. Interestingly, it has also been reported that human LC and dermal dendritic cells express hematopoietic PGD synthase (hPGDS) supporting these cells as a source of $\mathrm{PGD}_{2}$ in the skin, alongside mast cells and keratinocytes [19]. A role for $\mathrm{PGD}_{2}$ in photoimmunosuppression has not been explored but in murine skin and lung epithelia $\mathrm{PGD}_{2}$ inhibits dendritic cell migration and stimulation of $\mathrm{T}$ cell responses [20, 21]. The omega-3 polyunsaturated fatty acid (n-3 PUFA) eicosapentaenoic acid (EPA) reduces UVR-suppression of CMI in vivo; in mice, both topical and systemic EPA-rich lipids reduced UVR-suppression of chemically induced $\mathrm{CHS}$ responses by up to $90 \%$ [22, 23]. Further, we recently observed in a randomised controlled trial $(\mathrm{RCT})$ in humans that oral EPA supplementation showed potential to reduce UVR- UVR-suppression of nickel CHS [24]. While the mean group difference for the 3 solar simulated radiation (SSR) doses we employed showed no statistically significant protection by EPA, $50 \%$ reduction of photoimmunosuppression was noted with UVR dosing equivalent to brief exposure to summer sunlight (post-hoc analysis $p<0.05$ ) [24]. EPA exhibits a range of activities that may contribute to protective profile, including transcriptional activation of cytokine genes and modulation of PG synthesis [25]. EPA competes with the $n-6$ PUFA arachidonic acid (AA) for metabolism by cyclooxygenase (COX) enzymes, and this can reduce the levels of AAderived PG [26].

In a double-blind RCT in 79 females, the objective of the current study was to explore the impact of dietary EPA on epidermal LC numbers as a potential mechanism of abrogation of photoimmunosuppression, and to examine for influence on levels of immunomodulatory mediators. Cutaneous samples were taken from UVR-exposed and unexposed skin pre- and post- a 12-week course of supplementation, with immunofluorescence assessment of CD1a+ cells in epidermal sheets and quantification of PG and cytokines in blister fluid. 


\section{2}

\section{Materials and Methods}

\section{Participants}

Seventy-nine healthy female volunteers were recruited from the contact dermatitis investigation unit at Salford Royal Hospital, Manchester, UK and by open advertisement between 2008 and 2010. Inclusion criteria: age 18-60 years, female, Fitzpatrick sunreactive skin type I or II, allergic to nickel (required for the clinical photoimmunosuppression study, reported elsewhere [24]). Exclusion criteria: taking n-3 PUFA supplements or photoactive medication, pregnancy or breast feeding, sunbathing or sun bed use in the prior 3 months, history of photosensitivity, skin cancer or atopy. They did not have active contact dermatitis at the time of the study. Written informed consent was provided by all volunteers before study inclusion. Ethical approval was granted by North Manchester local research ethics committee $(08 / \mathrm{H} 1006 / 30)$ and the study was performed in accordance with the Declaration of Helsinki principles (revised Seoul 2008).

\section{Study Design and Intervention}

The double-blind randomised (1:1) controlled parallel-group study took place in the Photobiology Unit, Dermatology Centre, Salford Royal Hospital (Manchester, UK). Treatment allocation sequence was permuted block design (mixed blocks of 4 to 6 ) and produced by the study biostatistician using statistical software (v2.7.7; StatsDirect Ltd, Altrincham, UK). Encapsulated active and control lipid supplements, identical in appearance, were packaged and labeled according to the allocation sequence by GP solutions Ltd (Manchester, UK), and the code held by the study biostatistician until study completion. All volunteers and researchers were blinded and volunteers were assigned the intervention on study enrolment and concurrently randomised to have either suction blister fluid sampled for analysis of eicosanoids and cytokines or skin punch biopsies taken for assessment of epidermal LC. Skin sampling was performed on both unexposed and UVRexposed skin. All volunteers provided blood samples pre- and post-supplementation and 
compliance with supplementation was confirmed through measurement of red blood cell (RBC) EPA levels (reported in [24]). The parameters assessed here were secondary outcome measures in a larger clinical trial of oral EPA supplementation that primarily assessed impact on clinical photoimmunosuppression (nickel CHS; reported in [24]). Procedures in the different studies involved UVR-exposure to small skin areas only, at separate body sites and times, with the CHS study performed post-supplementation after completion of the current study. The n-3 PUFA supplements were $1 \mathrm{~g}$ gelatine capsules containing Incromega E7010 SR ethyl ester ( 70\% EPA and 10\% DHA; Croda Chemicals Leek Ltd, Staffordshire, UK). Control supplements comprised $1 \mathrm{~g}$ gelatine capsules of identical appearance containing glyceryl tricoprylate coprate (GTCC; Croda Chemicals Leek Ltd), a medium chain triglyceride found in coconut oil, and previously used as control oil in human supplement studies [27-29]. Both supplements were taken 5 capsules daily with breakfast for 12 weeks.

\section{UVR-exposure and Skin Sampling}

All volunteers were exposed to broadband UVR (270-400nm, peak 310nm; 44\% UVB, 56\% UVA, 1\% UVC; TL12, Philips GmbH, Hamburg, Germany or UV21, Waldmann Co., VSSchwenningen, Germany). Lamp irradiance was monitored during each exposure using radiometers (Medical Physics Department, Dryburn Hospital and Waldmann IL730A, International Light, Newburyport, USA) traceable to the UK National Physical Laboratory. The individual's minimal erythemal dose (MED) was determined on study enrolment. Pre and post-supplementation, upper buttock sites were exposed to $4 \mathrm{x}$ the individual's MED. After $24 \mathrm{~h}$, skin suction blistering and skin punch biopsy were performed from UVRexposed and unexposed sites (methods as described in [30]). The 4x MED dose was chosen to provide a sufficient challenge to produce quantifiable increases in cytokine and eicosanoid expression in human skin in vivo [31, 32]. 


\section{Epidermal Langerhans Cell Counting}

Skin punch biopsies $(5 \mathrm{~mm})$ from unexposed and UVR-exposed sites were immediately placed in 0.02M ethylene diamine tetra acetic acid (EDTA) in phosphate-buffered saline (PBS). After $2 \mathrm{~h}$ incubation at $37^{\circ} \mathrm{C}$, epidermis was carefully peeled from dermis using forceps. Epidermal sheets were washed in PBS, fixed in ice-cold acetone (20 minutes) and re-washed in PBS, prior to incubation with mouse CD1a monoclonal primary antibody (clone NA1/34; IgG2a (Dako, Stockport, UK)) diluted to $10 \mu \mathrm{g} / \mathrm{ml}$ in PBS $(0.1 \%$ bovine serum albumin (BSA; Sigma-Aldrich, MO, USA) and with fluorescein isothiocyanate (FITC) conjugated goat anti-mouse secondary antibody (Dako; $1 / 100$ in PBS $(0.1 \%$ BSA)), before mounting in Citifluor media (Citifluor, London, UK). LCs were counted using an Olympus Bx50 fluorescence microscope fitted with an eyepiece graticule at 40x magnification. Fifty fields per graticule were counted for each epidermal sheet.

\section{Suction Blister Fluid Prostaglandin Measurement}

Lipidomic analysis by mass spectrometry was performed as described previously [33, 34]. In summary, blister fluid eicosanoids $(50-200 \mu \mathrm{l})$ were extracted in methanol-water (15\% wt/wt) and internal standard $\mathrm{PGB}_{2}-\mathrm{d4}$ (40ng) (Cayman Chemicals, Ann Arbor, MI, USA) was added. The extract was acidified to $\mathrm{pH} 3.0$ and applied to preconditioned solid-phase extraction (SPE) cartridge (C18-E $500 \mathrm{mg}, 6 \mathrm{~mL}$ ) (Phenomenex, Macclesfield, UK) and eluted with methyl formate. Chromatographic analysis was performed on a C18 column (Luna, 5 $\mu \mathrm{m}$, 2.0mm, Phenomenex, Macclesfield, UK) using HPLC (Alliance 2695, Waters, Elstree, Hertfordshire, UK) coupled to a triple quadrupole mass spectrometer with electrospray ionisation (ESI) (Quattro Ultima, Waters). Multiple reaction monitoring transitions were used to assay for the presence of $\mathrm{PGD}_{2}(\mathrm{~m} / \mathrm{z} 351>271)$ and its metabolites $P G J_{2}, \Delta^{12}-P J_{2}(\mathrm{~m} / z 333>271)$ and $15-$ deoxy- ${ }^{\Delta 12,14} \mathrm{PGJ}_{2}(\mathrm{~m} / \mathrm{z} 315>271)$. Results are expressed as $\mathrm{pg} / \mu \mathrm{l}$ of blister fluid, based on calibration lines constructed from commercially available standards (Cayman Chemicals). 
191 Suction Blister Fluid Cytokine Measurement

192 A panel of cytokines (IL-8, IFN- $\gamma$, TNF- $\alpha$, IL-1 $\beta$, IL-4, IL-10, IL-23 and IL-17) was

193 simultaneously quantified in suction blister fluid using the Bio-Plex ${ }^{\mathrm{TM}}$ cytokine array system

194 (Bio-Rad Laboratories, Hercules, CA, USA) in accordance with manufacturer's instructions,

195 as described previously [35].

\section{Statistical analysis}

197 The study was powered to detect a difference in clinical photoimmunosuppression

198 responses between EPA and control supplemented groups, as previously detailed [24].

199 Statistical analysis was performed in SPSS 20.0. Non-normally distributed data was

200 transformed using natural log. ANCOVA analyses compared EPA and control groups post-

201 supplementation with baseline (pre-supplementation) data as the covariate. Paired t-tests

202 were performed to make within-group comparisons between unexposed and UVR-exposed

203 skin. A $p$ value of $<0.05$ was considered statistically significant.

204

205

206

207

208

209

210

211

212

213

214

215

216

217 
218 Results

219 Volunteers and compliance

220 Seventy-nine volunteers were recruited and randomised to the oral intervention: 6 did not

221 proceed to take supplements and discontinued the study for personal reasons; no data was

222 collected from them. Of the 73 who took supplements, 33 were randomised to control and

22340 to EPA; baseline characteristics are shown (Table 1). Baseline dietary intake assessed

224 by food frequency questionnaire was below current UK recommendations of $450 \mathrm{mg} /$ day total

225 long chain n-3 PUFA [36, 37]. The EPA supplement was bioavailable in both RBC and skin

$226(p<0.001)$ as previously reported [30]. Three volunteers in the EPA group (all suction blister

227 subgroup) who showed no increase in RBC EPA levels post-supplementation were excluded

228 from analyses for poor compliance (Fig 1). One individual in the EPA group declined

229 biopsies post-supplementation and data was excluded from analyses. Of the remaining 69

230 volunteers, 33 were in the control and 36 in the EPA group (Fig 1). No adverse effects were

231 reported for either supplement.

232

\section{Langerhans cells}

234 To assess the effect of UVR exposure on epidermal LC density pre-supplementation, 235 baseline data of the two supplement groups was combined. UVR challenge produced a 236 reduction of $\sim 65 \%$ in mean (SEM) LC number in the epidermis at 24 hours post-exposure,

237 from 920 (28) to 318 (39) per $\mathrm{mm}^{2}(p<0.001)$ (Fig 2A). Following supplementation, the UVR-

238 induced reduction in LC number was similar to baseline for both control (881 (46) to 218 (44)

239 cells per $\mathrm{mm}^{2} ; p<0.001$ ) and EPA group (856 (55) to 191 (26) cells per $\mathrm{mm}^{2} ; p<0.001$ ),

240 decreases of $75 \%$ and $78 \%$, respectively (Fig $2 \mathrm{~A}$ ). There was no significant difference in

241 epidermal LC numbers between control and EPA groups post-supplementation, in

242 unexposed or UVR-exposed skin. Visualisation of LC in epidermal sheets revealed that

243 following UVR the majority of LC lost their dendritic projections and appeared in a more

244 rounded, migratory form. There was no apparent effect of EPA on LC morphology (Fig 2B) in

245 unexposed or UVR-exposed skin. 


\section{Prostaglandin production}

$248 \mathrm{PGD}_{2}$ and its metabolites $P G J_{2}, \Delta^{12}-\mathrm{PGJ}_{2}$ and 15-deoxy- ${ }^{\Delta 12,14} P \mathrm{PJ}_{2}$ were measured in skin

249 blister fluid to explore impact of UVR and EPA; $\Delta^{12}-\mathrm{PGJ}_{2}$ was detected but below the limit of

250 quantitation and 15-deoxy- ${ }^{{ }^{12,14}} \mathrm{PGJ}_{2}$ was below limit of detection. At baseline, data from

251 both supplement groups was combined to examine effect of UVR exposure.

252

253

$P G D_{2}$ : At baseline, median (IQR) $P G D_{2}$ was decreased in UVR-exposed versus unexposed

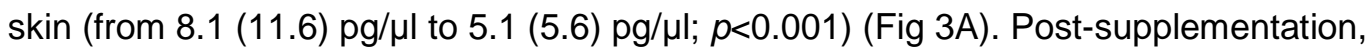

control group $\mathrm{PGD}_{2}$ level was similarly decreased in UVR-exposed versus unexposed skin

(from $8.6(6.3)$ to $4.1(4.7) \mathrm{pg} / \mu \mathrm{l} ; p<0.01)$. In contrast in the EPA group post-

supplementation, no statistically significant reduction in $\mathrm{PGD}_{2}$ occurred post-UVR.

Comparison of groups post-supplementation revealed that in unexposed skin $\mathrm{PGD}_{2}$ was

$\sim 40 \%$ lower in the EPA-unexposed versus control group (5.2 (4.8) vs 8.6 (6.3) noncontrol group).

$P G J_{2}$ : At baseline, $P G J_{2}$ was significantly increased in UVR-exposed versus unexposed skin in $P G J_{2}$ were seen in UVR-exposed skin in control and EPA groups (non-significant). There were no significant differences in $P G J_{2}$ levels between control and EPA groups postsupplementation.

\section{Cytokine expression}

270 Of the panel of cytokines assessed, IL-10, TNF $\alpha$ and IL-8 were quantifiable. Whilst IFN- $\gamma$ was detected, levels were below the limit of quantitation, and IL-1 $\beta, I L-4, I L-17$ and IL-23 were not detected. Due to low blister fluid volumes, five individuals (two in EPA group and

273 three in control group) were excluded from cytokine analyses, resulting in $n=16$ for the 
274 control and $n=15$ for the EPA group. IL-10 levels for two individuals in the control group were

275 out of range and excluded, resulting in $n=14$ in the control group. Baseline data for EPA and

276 control groups were combined to assess effect of UVR on cytokine levels pre-

277 supplementation.

278

279 IL-8: At baseline, median (IQR) IL-8 increased in UVR-exposed versus unexposed skin

280 (791.9 (798.9) vs 238.1 (314) pg/ml; p<0.001; Fig 3D). Similarly, post-supplementation, a

281 statistically significant UVR-induced rise in IL-8 was seen in the control group (from 162.3

282 (304.2) to $827.1(443) \mathrm{pg} / \mathrm{ml} ; p<0.001)$ and EPA group (from 244.5 (277.3) to 591.7 (970.9)

$283 \mathrm{pg} / \mathrm{ml} ; p<0.01)$. There was no significant difference in IL-8 concentration in unexposed or

284 UVR-exposed skin in control versus treatment groups post-supplementation.

285

286

287

IL-10: At baseline, median (IQR) IL-10 concentration apparently increased following UVR exposure, but this was not statistically significant (82 (153) vs 68.3 (142) pg/ml; Fig 3E).

Similarly post-supplementation, there was an apparent increase in IL-10 concentration postUVR in the control (90.3 (142) vs 79.6 (98) pg/ml) and EPA groups (95.8 (148) vs 70 (115) $\mathrm{pg} / \mathrm{ml}$ ). There was no significant difference in IL-10 concentration in unexposed or UVRexposed skin when comparing control and EPA groups post-supplementation.

292

TNF $\alpha$ : At baseline, median (IQR) TNF $\alpha$ concentration was not significantly altered in UVRexposed versus unexposed skin (67.2 (98.5) pg/ml vs $57.7(101.8) \mathrm{pg} / \mathrm{ml})$ at baseline (Fig 3F). Post-supplementation, there were apparent rises in TNF $\alpha$ in UVR-exposed versus unexposed skin, in control (84.8 (107.2) vs 54.7 (139.7)) and EPA (88.1 (149.9) vs 36.6 (66.2)) groups (both non-significant). There was no significant difference in TNF $\alpha$ concentration in unexposed or UVR-exposed skin when comparing control and EPA groups post-supplementation. 
301

302

303

304

305

306

307

308

309

310

311

312

313

\section{Discussion}

In this study UVR exposure of human skin in vivo at baseline (pre-supplementation)

significantly reduced epidermal LC density and altered the morphology of remaining LC, in association with a notable reduction in $P G D_{2}$. This significant UVR impact on $P G D_{2}$ production $(\mathrm{Fig} 3 \mathrm{~A})$ is in stark contrast to the well-described increase in skin $\mathrm{PGE}_{2}$ and other eicosanoids examined following UVR-exposure to humans in vivo [30, 31], and this could have implications for health, including during sun-exposure and for the phototherapy of skin disorders. The subsequent investigation of the impact of 12 weeks EPA supplementation employed a robust study design and adequate sample size, and importantly, oral EPA compliance and skin bioavailability was demonstrated in these volunteers [24, 30]. No impact of EPA supplementation versus control was found on epidermal LC numbers, either in unexposed or UVR-exposed skin, and hence we found no evidence that EPA abrogates UVR-suppression of skin immunity through this mechanism in humans.

UVR induced loss of LC from the epidermis contributes to local UVR-induced immunosuppression of the skin, which is partially mediated through induction of T-reg [11]. Langerhans cell loss from the epidermis can be stimulated by a range of UVR doses, with LC cell density and size reduction occurring in a UVR-dose dependent manner [38, 39]. We observed a notable reduction in epidermal LC number of $\sim 65 \%$ following a pro-inflammatory (4XMED) UVR exposure. This magnitude of response is in line with a previous report in human skin, where LC apoptosis in the epidermis was barely detectable after a very high (6xMED) UVR challenge, while migration was observed [6], supporting that the UVR-induced epidermal loss of LC observed in our study could be due to migration. The current study provides substantially the largest dataset to-date examining UVR-induced reduction in LC number in human epidermis. Consistent with previous observations [40, 41], notable intersubject variation was seen in LC numbers under all treatment conditions.

While most skin blister fluid cytokines assessed in our study were below the assay detection limit, the chemokine IL-8 showed a large induction in response to UVR-exposure, in keeping with previous studies [16, 32]. TNF- $\alpha$ and IL- $\beta$ are key cytokines involved in LC 
329 mobilisation following exposure to UVR [13, 42], however, IL-1 $\beta$ was not detected and while

330 TNF- $\alpha$ was present, no significant UVR-induced increase was found in blister fluid. A major

331 source of UVR-induced TNF- $\alpha$ is purported to be basal keratinocytes [43], where UVR-

332 induced nuclear DNA damage may stimulate its release [44]. The dermal neutrophil infiltrate,

333 which is reported to peak from 14 hours post-UVR may also contribute to TNF $\alpha$ increase

334 [45]. The immunosuppressive cytokine IL-10 inhibits dendritic cell IFN- $\gamma$ production and

335 initiation of CMI responses and induces tolerance [46-48]. In UVR-irradiated human skin, IL-

33610 is reported to be preferentially induced in infiltrating CD11b+ macrophages which peak in

337 the dermis during the first 24 hours and in epidermis at 72 hours [49]; as blister fluid is

338 primarily of epidermal origin [34] this might contribute to lack of significant IL-10 increase

339 observed in this study. No effect of EPA on cytokine levels was observed. A 24 hour post-

340 UVR time point was selected as the most appropriate for assessing cytokines and

341 prostaglandins simultaneously [16, 31], however, other time points might reveal differences.

342 We previously reported the skin $\mathrm{PGE}_{2}$ level in this group of individuals was

343 augmented at 24 hours post UVR challenge [30]. PGE 2 stimulates IL-10 production in mouse

344 and human model systems, favouring a Th2 response, Treg activation and immune-

345 suppression $[15,50,51]$. In contrast to the $127 \%$ rise in $P_{G E}$, we found skin $P D_{2}$ levels in

346 the same volunteers were significantly reduced by $37 \%$ after UVR-exposure (Fig $3 C)$. $P \mathrm{CD}_{2}$

347 is associated with allergic inflammatory disorders in the respiratory tract [52] and skin [53],

348 including mast cell disorders [54] and atopic dermatitis [55, 56], and has potential relevance

349 to the novel treatment of other conditions featuring raised cutaneous $\mathrm{PGD}_{2}$, including hair-

350 loss [57]. $\mathrm{PGD}_{2}$ differentially regulates $\mathrm{T}$ cell responses via two receptors; the DP1 receptor

351 mediates inhibition of Th1 functions, while the DP2 (CRTH2) receptor promotes Th2 activity

352 [58]. In inflammatory skin disorders the contrasting effect of acute UVR exposure on $\mathrm{PGD}_{2}$

353 and $\mathrm{PGE}_{2}$ may contribute to the therapeutic effects of phototherapy. Increased levels of the

$354 \mathrm{PGD}_{2}$ dehydration product $\mathrm{PGJ}_{22}$ in UVR-exposed skin is also interestingly, as $\mathrm{J}$ ring 
355

356

357

358

metabolites, in particular $15-$ deoxy- $\Delta^{12,14}-\mathrm{PGJ}_{2}$, reportedly exert anti-inflammatory effects [59]. Further assessment of these metabolites in cutaneous inflammation could be valuable. In human skin, LC, mast cells and dermal dendritic cells are primary sources of $\mathrm{PGD}_{2}$ [19]. Post UVR-exposure of human skin-mast cell infiltration and degranulation occurs as early as 4 hours post-challenge, but by 24 hours mast cell numbers and activity have returned to normal [60], while epidermal LC are depleted. We propose that the UVRreduction in cutaneous $P G D_{2}$ could partially reflect loss of $L C$ from the epidermis. This is supported by our observation of no UVR-induced $\mathrm{PGD}_{2}$ reduction in human primary keratinocytes and fibroblasts (unpublished data), or in the dermal fraction of human skin [61]. In mice ageing-associated increases in local $\mathrm{PGD}_{2}$ correlate with impaired migration of respiratory DC, and antagonism of the DP1 receptor restores migration [16]. Constitutive levels of $\mathrm{PGD}_{2}$ may provide an inhibitory signal to migration which can be downregulated by UVR. While we did not find an effect of EPA on PGD 2 levels in UVR-exposed skin, an apparent fall in unexposed skin compared to control (Fig 3A) was consistent with in vitro findings of EPA reduction of $\mathrm{PGD}_{2}$ production in niacin- stimulated human $\mathrm{LC}$ [62].

Our recently reported assessment of a clinical CHS end-point in the same volunteers suggested that 12 weeks oral EPA supplementation has the potential to reduce UVRsuppression of nickel CHS [24]. However, we have found EPA to have no impact on LC number in unexposed and UVR-exposed skin when compared to the control group, when using a high UVR-dose sufficient to produce a measurable increase in PG [30,31] Thus, our study did not support the mediation of immune-protective effects of EPA by changes in the numbers of epidermal LC, although it is conceivable there may still have been changes in LC activity. EPA may potentially exhibit greater protection with UVR at lower doses or different spectra. This could be addressed in future studies, alongside examination of impact on other DC subsets as understanding of their significance in human skin immunity becomes better understood.

In conclusion, our double blind RCT did not find evidence for an impact of oral EPA on UVR-induced reduction of epidermal LC. The significant UVR-induced fall in $\mathrm{PGD}_{2}$ level 
383 may have an immunomodulatory effect of relevance to the phototherapy of skin disease, and 384 warrants further investigation.

385

386 Acknowledgements

387 LER, NKG and PSF designed the study, SMP, PC, SPB and KAM performed the study and 388 data analysis, AN contributed essential equipment and reagents, SMP and LER wrote the 389 paper and PSF and AN critically revised the paper. We acknowledge the Association of 390 International Cancer Research for funding this study. We thank Rebecca Dearman and Ian 391 Kimber in the Faculty of Life Sciences at the University of Manchester for use of their 392 Luminex analyser, Donald Allan for technical support, Croda Chemicals Ltd for freely 393 supplying the active and control lipid supplements and GP solutions Ltd for packaging the 394 supplements. We also thank all the volunteers who took part in the study.

395

396 Conflict of Interests

397 The authors have no conflicts of interest. 


\section{References}

399 1. Schwarz, T., Photoimmunosuppression. Photodermatol Photoimmunol Photomed 400 2002: 18: 141-145.

4012 Kripke, M. L., R. M. Thorn, P. H. Lill, C. I. Civin, N. H. PazmiñoM. S. Fisher, Further

402 characterization of immunological unresponsiveness induced in mice by ultraviolet radiation.

403 Growth and induction of nonultraviolet-induced tumors in ultraviolet-irradiated mice.

404 Transplantation 1979: 28: 212-217.

$4053 . \quad$ Oberyszyn, T. M., Non-melanoma skin cancer: importance of gender, 406 immunosuppressive status and vitamin D. Cancer Lett 2008: 261: 127-136.

407 4. van der Aar, A. M., D. I. Picavet, F. J. Muller et al., Langerhans cells favor skin flora 408 tolerance through limited presentation of bacterial antigens and induction of regulatory $T$ 409 cells. J Invest Dermatol 2013: 133: 1240-1249.

410 5. Seneschal, J., Rachael A. Clark, A. Gehad, Clare M. Baecher-AllanThomas S.

411 Kupper, Human Epidermal Langerhans Cells Maintain Immune Homeostasis in Skin by

412 Activating Skin Resident Regulatory T Cells. Immunity 2012: 36: 873-884.

413 6. Kolgen, W., H. Both, H. van Weelden et al., Epidermal Langerhans Cell Depletion 414 After Artificial Ultraviolet B Irradiation of Human Skin In Vivo: Apoptosis Versus Migration. J 415 Invest Dermatol 2002: 118: 812-817.

$4167 . \quad$ Simon, J. C., P. D. Cruz, Jr., P. R. BergstresserR. E. Tigelaar, Low dose ultraviolet

417 B-irradiated Langerhans cells preferentially activate CD4+ cells of the T helper 2 subset. J 418 Immunol 1990: 145: 2087-2091.

419 8. Simon, J. C., R. E. Tigelaar, P. R. Bergstresser, D. EdelbaumP. D. Cruz, Jr., 420 Ultraviolet B radiation converts Langerhans cells from immunogenic to tolerogenic antigen421 presenting cells. Induction of specific clonal anergy in CD4+ T helper 1 cells. J Immunol 422 1991: 146: 485-491.

4239 9. Loser, K.S. Beissert, Regulation of cutaneous immunity by the environment: an 424 important role for UV irradiation and vitamin D. Int Immunopharmacol 2009: 9: 587-589. 
425 10. Denfeld, R. W., H. Hara, J. P. Tesmann, S. MartinJ. C. Simon, UVB-irradiated

426 dendritic cells are impaired in their APC function and tolerize primed Th1 cells but not naive

427 CD4+ T cells. J Leukoc Biol 2001: 69: 548-554.

428 11. Schwarz, A., M. Noordegraaf, A. Maeda, K. Torii, B. E. ClausenT. Schwarz,

429 Langerhans cells are required for UVR-induced immunosuppression. J Invest Dermatol

430 2010: 130: 1419-1427.

431 12. Damian, D. L.G. M. Halliday, Measurement of ultraviolet radiation-induced

432 suppression of recall contact and delayed-type hypersensitivity in humans. Methods 2002:

$433 \quad 28: 34-45$.

434 13. Cumberbatch, M., R. J. Dearmanl. Kimber, Interleukin 1 beta and the stimulation of

435 Langerhans cell migration: comparisons with tumour necrosis factor alpha. Arch Dermatol

436 Res 1997: 289: 277-284.

437 14. Cumberbatch, M., I. Fieldingl. Kimber, Modulation of epidermal Langerhans' cell

438 frequency by tumour necrosis factor-alpha. Immunology 1994: 81: 395-401.

439 15. Shreedhar, V., T. Giese, V. W. SungS. E. Ullrich, A cytokine cascade including

440 prostaglandin E2, IL-4, and IL-10 is responsible for UV-induced systemic immune

441 suppression. J Immunol 1998: 160: 3783-3789.

442 16. Strickland, I., L. E. Rhodes, B. F. FlanaganP. S. Friedmann, TNF-alpha and IL-8 are

443 upregulated in the epidermis of normal human skin after UVB exposure: correlation with

444 neutrophil accumulation and E-selectin expression. J Invest Dermatol 1997: 108: 763-768.

445 17. Kabashima, K., D. Sakata, M. Nagamachi, Y. Miyachi, K. InabaS. Narumiya,

446 Prostaglandin E2-EP4 signaling initiates skin immune responses by promoting migration and

447 maturation of Langerhans cells. Nat Med 2003: 9: 744-749.

448 18. Ng, R. L., J. L. Bisley, S. Gorman, M. NorvalP. H. Hart, Ultraviolet irradiation of mice

449 reduces the competency of bone marrow-derived CD11c+ cells via an indomethacin-

450 inhibitable pathway. J Immunol 2010: 185: 7207-7215. 
451 19. Shimura, C., T. Satoh, K. Igawa et al., Dendritic Cells Express Hematopoietic

452 Prostaglandin D Synthase and Function as a Source of Prostaglandin D2 in the Skin. Am J

453 Pathol 2010: 176: 227-237.

454 20. Zhao, J., J. Zhao, K. LeggeS. Perlman, Age-related increases in PGD(2) expression

455 impair respiratory DC migration, resulting in diminished T cell responses upon respiratory

456 virus infection in mice. J Clin Invest 2011: 121: 4921-4930.

457 21. Angeli, V., C. Faveeuw, O. Roye et al., Role of the parasite-derived prostaglandin D2

458 in the inhibition of epidermal Langerhans cell migration during schistosomiasis infection. J

459 Exp Med 2001: 193: 1135-1147.

460 22. Moison, R. M.G. M. Beijersbergen Van Henegouwen, Dietary eicosapentaenoic acid

461 prevents systemic immunosuppression in mice induced by UVB radiation. Radiat Res 2001:

462 156: 36-44.

463 23. Moison, R. M., D. P. SteenvoordenG. M. Beijersbergen van Henegouwen, Topically

464 applied eicosapentaenoic acid protects against local immunosuppression induced by UVB

465 irradiation, cis-urocanic acid and thymidine dinucleotides. Photochem Photobiol 2001: 73:

$466 \quad 64-70$.

467 24. Pilkington, S. M., K. A. Massey, S. P. Bennett et al., Randomized controlled trial of

468 oral omega-3 PUFA in solar-simulated radiation-induced suppression of human cutaneous

469 immune responses. Am J Clin Nutr 2013: 97: 646-652.

470 25. Pilkington, S. M., R. E. Watson, A. NicolaouL. E. Rhodes, Omega-3 polyunsaturated

471 fatty acids: photoprotective macronutrients. Exp Derm 2011: 20: 537-543.

472 26. Wada, M., C. J. DeLong, Y. H. Hong et al., Enzymes and Receptors of Prostaglandin

473 Pathways with Arachidonic Acid-derived Versus Eicosapentaenoic Acid-derived Substrates

474 and Products. J Biol Chem 2007: 282: 22254-22266.

475 27. West, N. J., S. K. Clark, R. K. Phillips et al., Eicosapentaenoic acid reduces rectal

476 polyp number and size in familial adenomatous polyposis. Gut 2010: 59: 918-925. 
477 28. Belluzzi, A., C. Brignola, M. Campieri, A. Pera, S. BoschiM. Miglioli, Effect of an

478 enteric-coated fish-oil preparation on relapses in Crohn's disease. N Engl J Med 1996: 334:

$479 \quad 1557-1560$.

480 29. Henz, B. M., S. Jablonska, P. C. van de Kerkhof et al., Double-blind, multicentre

481 analysis of the efficacy of borage oil in patients with atopic eczema. Br J Dermatol 1999:

482 140: 685-688.

483 30. Pilkington, S. M., L. E. Rhodes, N. M. Al-Aasswad, K. A. MasseyA. Nicolaou, Impact

484 of EPA ingestion on COX- and LOX-mediated eicosanoid synthesis in skin with and without

485 a pro-inflammatory UVR challenge--report of a randomised controlled study in humans. Mol

$486 \quad$ Nutr Food Res 2014: 58: 580-590.

487 31. Rhodes, L. E., K. Gledhill, M. Masoodi et al., The sunburn response in human skin is

488 characterized by sequential eicosanoid profiles that may mediate its early and late phases.

489 FASEB J. 2009: 23: 3947-3956.

490 32. Shahbakhti, H., R. E. Watson, R. M. Azurdia, C. Z. Ferreira, M. GarmynL. E. Rhodes,

491 Influence of eicosapentaenoic acid, an omega-3 fatty acid, on ultraviolet-B generation of

492 prostaglandin-E2 and proinflammatory cytokines interleukin-1 beta, tumor necrosis factor-

493 alpha, interleukin-6 and interleukin-8 in human skin in vivo. Photochem Photobiol 2004: 80:

$494 \quad 231-235$.

495 33. Masoodi, M.A. Nicolaou, Lipidomic analysis of twenty-seven prostanoids and

496 isoprostanes by liquid chromatography/electrospray tandem mass spectrometry. Rapid

497 Commun Mass Spectrom 2006: 20: 3023-3029.

498 34. Kendall, A. C., S. M. Pilkington, K. A. Massey, G. Sassano, L. E. RhodesA. Nicolaou,

499 Distribution of bioactive lipid mediators in human skin. J Invest Dermatol 2015: 135: 1510-

5001520.

501 35. Dearman, R. J., C. J. Betts, H. T. Caddickl. Kimber, Cytokine profiling of chemical

502 allergens in mice: impact of mitogen on selectivity of response. J Appl Toxicol 2009: 29: 233-

503241. 
504 36. Scientific Advisory Committee on Nutrition (SACN), Advice on fish consumption:

505 benefits and risks. 2004, London: The Stationary Office.

506 37. Wallingford, S. C., S. M. Pilkington, K. A. Massey et al., Three-way assessment of

507 long chain omega-3 polyunsaturated fatty acid nutrition: by questionnaire and matched blood 508 and skin samples. Br J Nutr 2012: 23: 1-8.

509 38. Blackburn, A., T. C. Ling, M. Brownrigg, L. E. RhodesN. K. Gibbs, UVB-induced 510 Langerhans cell trafficking in polymorphic light eruption. Br J Dermatol 2004: 150: 796.

511 39. Seite, S., H. Zucchi, D. Moyal et al., Alterations in human epidermal Langerhans cells 512 by ultraviolet radiation: quantitative and morphological study. $\mathrm{Br} J$ Dermatol 2003: 148: 291513299.

514 40. Cumberbatch, M., M. Singh, R. J. Dearman, H. S. Young, I. KimberC. E. Griffiths, 515 Impaired Langerhans cell migration in psoriasis. J Exp Med 2006: 203: 953-960.

516 41. Cumberbatch, M., M. Bhushan, R. J. Dearman, I. KimberC. E. Griffiths, IL-1beta517 induced Langerhans' cell migration and TNF-alpha production in human skin: regulation by 518 lactoferrin. Clin Exp Immunol 2003: 132: 352-359.

519 42. Cumberbatch, M., R. J. Dearmanl. Kimber, Langerhans cells require signals from 520 both tumour necrosis factor-alpha and interleukin-1 beta for migration. Immunology 1997: 521 92: 388-395.

522 43. Human keratinocytes are a source for tumor necrosis factor alpha: evidence for 523 synthesis and release upon stimulation with endotoxin or ultraviolet light. J Exp Med 1990: 524 172: 1609-1614.

525 44. Walker, S. L.A. R. Young, An action spectrum $(290-320 \mathrm{~nm})$ for TNFalpha protein in 526 human skin in vivo suggests that basal-layer epidermal DNA is the chromophore. Proc Natl 527 Acad Sci U S A 2007: 104: 19051-19054.

528 45. Hawk, J. L., G. M. MurphyC. A. Holden, The presence of neutrophils in human 529 cutaneous ultraviolet-B inflammation. Br J Dermatol 1988: 118: 27-30. 
530 46. Ullrich, S. E., Mechanism involved in the systemic suppression of antigen-presenting 531 cell function by UV irradiation. Keratinocyte-derived IL-10 modulates antigen-presenting cell 532 function of splenic adherent cells. J Immunol 1994: 152: 3410-3416.

533 47. Schwarz, A., S. Grabbe, H. Riemann et al., In vivo effects of interleukin-10 on contact 534 hypersensitivity and delayed-type hypersensitivity reactions. J Invest Dermatol 1994: 103: $535 \quad 211-216$.

536 48. Enk, A. H., J. Saloga, D. Becker, M. MohamadzadehJ. Knop, Induction of hapten537 specific tolerance by interleukin 10 in vivo. J Exp Med 1994: 179: 1397-1402.

538 49. Kang, K., A. C. Gilliam, G. Chen, E. TootellK. D. Cooper, In human skin, UVB 539 initiates early induction of IL-10 over IL-12 preferentially in the expanding dermal 540 monocytic/macrophagic population. J Invest Dermatol 1998: 111:31-38.

541 50. Kalinski, P., C. M. Hilkens, A. Snijders, F. G. SnijdewintM. L. Kapsenberg, Dendritic 542 cells, obtained from peripheral blood precursors in the presence of PGE2, promote Th2 543 responses. Adv Exp Med Biol 1997: 417: 363-367.

544 51. Harizi, H., M. Juzan, V. Pitard, J. F. MoreauN. Gualde, Cyclooxygenase-2-issued 545 prostaglandin e(2) enhances the production of endogenous IL-10, which down-regulates 546 dendritic cell functions. J Immunol 2002: 168: 2255-2263.

547 52. García-Solaesa, V., C. Sanz-Lozano, J. Padrón-Morales et al., The prostaglandin D2 548 receptor (PTGDR) gene in asthma and allergic diseases. Allergol Immunopathol 2014: 42: $549 \quad 64-68$.

550 53. Yahara, H., T. Satoh, C. MiyagishiH. Yokozeki, Increased expression of CRTH2 on 551 eosinophils in allergic skin diseases. J Eur Acad Dermatol Venereol 2010: 24: 75-76.

552 54. Butterfield, J. H.C. R. Weiler, Prevention of mast cell activation disorder-associated 553 clinical sequelae of excessive prostaglandin $\mathrm{D}(2)$ production. Int Archives Allergy Immunol 554 2008: 147: 338-343.

555 55. Matsushima, Y., T. Satoh, Y. Yamamoto, M. NakamuraH. Yokozeki, Distinct roles of 556 prostaglandin D2 receptors in chronic skin inflammation. Mol Immunol 2011: 49: 304-310. 
557 56. Iwasaki, M., K. Nagata, S. Takano, K. Takahashi, N. IshiiZ. Ikezawa, Association of a 558 new-type prostaglandin D2 receptor CRTH2 with circulating T helper 2 cells in patients with 559 atopic dermatitis. J Invest Dermatol 2002: 119: 609-616.

560 57. Nieves, A.L. A. Garza, Does Prostaglandin D(2) hold the cure to male pattern 561 baldness? Exp Dermatol 2014: 23: 224-227.

562 58. Tanaka, K., H. Hirai, S. Takano, M. NakamuraK. Nagata, Effects of prostaglandin D2 563 on helper T cell functions. Biochem Biophys Res Commun 2004: 316: 1009-1014.

564 59. Harris, S. G., J. Padilla, L. Koumas, D. RayR. P. Phipps, Prostaglandins as 565 modulators of immunity. Trends Immunol 2002: 23: 144-150.

566 60. Gilchrest, B. A., N. A. Soter, J. S. StoffM. C. Mihm, Jr., The human sunburn reaction: 567 histologic and biochemical studies. J Am Acad Dermatol 1981: 5: 411-422.

568 61. Kendall, A. C., S. M. Pilkington, G. Sassano, L. E. RhodesA. Nicolaou, N-acyl 569 ethanolamide and eicosanoid involvement in irritant dermatitis. Br J Dermatol 2016.

570 62. VanHorn, J., J. D. Altenburg, K. A. Harvey, Z. Xu, R. J. KovacsR. A. Siddiqui, 571 Attenuation of niacin-induced prostaglandin $\mathrm{D}(2)$ generation by omega-3 fatty acids in THP-1 572 macrophages and Langerhans dendritic cells. J Inflamm Res 2012: 5: 37-50.

573 63. Judson, B. L., A. Miyaki, V. D. Kekatpure et al., UV Radiation Inhibits 15-

574 Hydroxyprostaglandin Dehydrogenase Levels in Human Skin: Evidence of Transcriptional 575 Suppression. Cancer Prev Res 2010: 3: 1104-1111.

576 64. Kalinski, P., J. H. Schuitemaker, C. M. HilkensM. L. Kapsenberg, Prostaglandin E2 577 induces the final maturation of IL-12-deficient CD1a+CD83+ dendritic cells: the levels of IL57812 are determined during the final dendritic cell maturation and are resistant to further 579 modulation. J Immunol 1998: 161: 2804-2809. 
583 Table 1. Baseline characteristics of participants.

584

\begin{tabular}{lcc}
\hline Characteristic & Control & EPA \\
\hline Age (yrs) (median (range)) & $45(22-60)$ & $43(21-60)$ \\
$\mathrm{BMI}^{1}\left(\mathrm{Kg} / \mathrm{m}^{2}\right)($ mean (SD)) & $25.9(4.4)$ & $27.8(5.3)$ \\
Skin type & \\
$I$ & & $6 / 40(15)$ \\
$I I$ & $2 / 33(6)$ & $34 / 40(85)$ \\
$\mathrm{HRT} / \mathrm{OCP}^{3}$ (no./total (\%)) & $31 / 33(94)$ & $6 / 40(15)$
\end{tabular}

$585{ }^{1}$ BMI data from $n=31$ in the EPA group and $n=31$ in the control group

$586{ }^{2}$ Fitzpatrick skin type classification: I- always burns, never tans, II- usually burns, tans with

587 difficulty

$588{ }^{3}$ Hormone replacement therapy/ oral contraceptive pill

589

590

591 
592 Figure legends

593

594 Fig 1. Flow diagram of study design and participants.

595

596 Fig 2. UVR induces LC loss from epidermis but EPA supplementation has no impact

597 on epidermal LC numbers. (A) LC count (mean) per $\mathrm{mm}^{2}$ of epidermis and (B) images of

598 CD1a positive LC in epidermal sheets in unexposed (open circles) and UVR-exposed skin

599 (closed circles) at baseline $(n=30)$ and post-supplementation (control $n=12$, EPA $n=18$ );

$600{ }^{* * *} p<0.001$ (scale bar $\left.50 \mu \mathrm{m}\right)$.

601

602 Fig 3. UVR reduces $\mathrm{PGD}_{2}$ and increases IL-8 level in skin blister fluid.

603 Concentration (median) of $(A) P G D_{2}$, and $(B)$ its metabolite $P G J_{2}$ in skin blister fluid taken

604 from unexposed (open circles) and UVR-exposed (closed circles) skin at baseline ( $\mathrm{n}=36)$

605 and post-supplementation (control $n=19$, EPA $n=17$ ); ${ }^{*} p<0.05$, ${ }^{* *} p<0.01$. (C) \% change in

$606 \mathrm{PGD}_{2}$ in UVR-exposed skin in comparison with UVR-induced \% change in $\mathrm{PGE}_{2}$ [24], in skin

607 blister fluid at baseline $(n=36)$. Concentration of (D) IL-8, (E) IL-10 and (F) TNF- $\alpha$ in skin

608 blister fluid taken from unexposed (open circles) and UVR-exposed (closed circles) skin at

609 baseline $(n=31)$ and post-supplementation (control $n=16(\operatorname{IL}-10 n=14)$, EPA $n=15) ;{ }^{*} p<0.05$,

$610{ }^{* *} p<0.01,{ }^{* * *} p<0.001$.

611

612

613

614

615

616

617

618

619 
Fig 1.

621

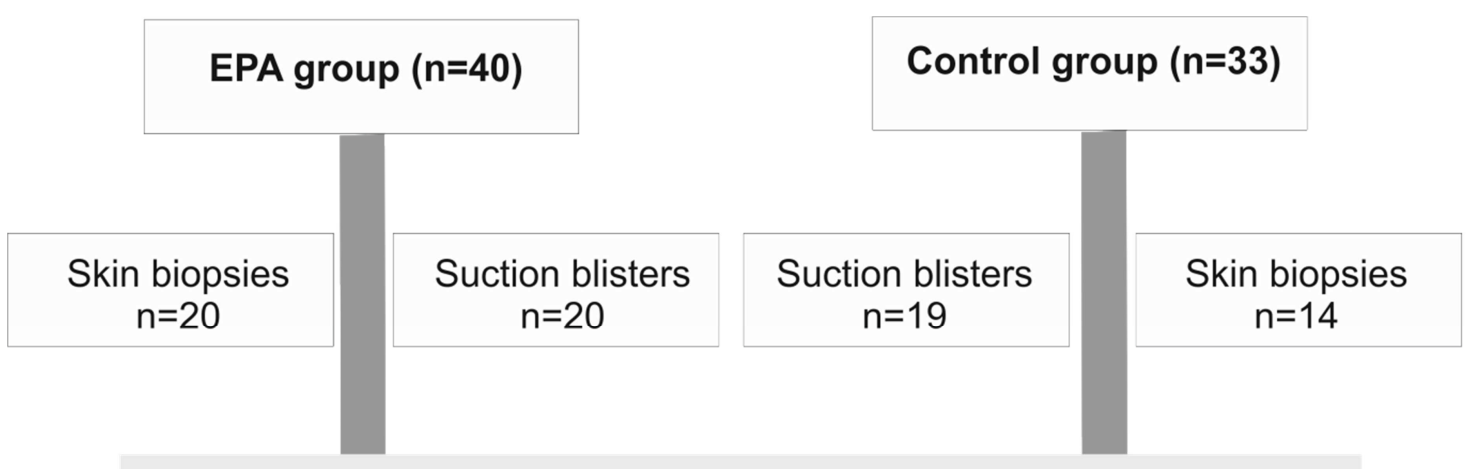

12 weeks supplementation with EPA or control lipid

Excluded for poor compliance $n=3$

Damaged epidermal sheets $n=1$

Skin biopsies declined; data excluded $n=1$

Insufficient suction blister volume for cytokines $n=2$

\section{LC counts $n=18$}

Cytokine analyses $n=15$

Eicosanoid analyses $n=17$
Damaged epidermal sheets $n=2$

Insufficient suction blister volume for cytokines $n=3$

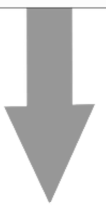

LC counts $n=12$

Cytokine analyses $n=16$

Eicosanoid analyses $n=19$ 
641

642

643

644

645

646

647

648

649

650

651

652

653

654

655

656

657

658

659

660

661

662

663
Fig 2.

A

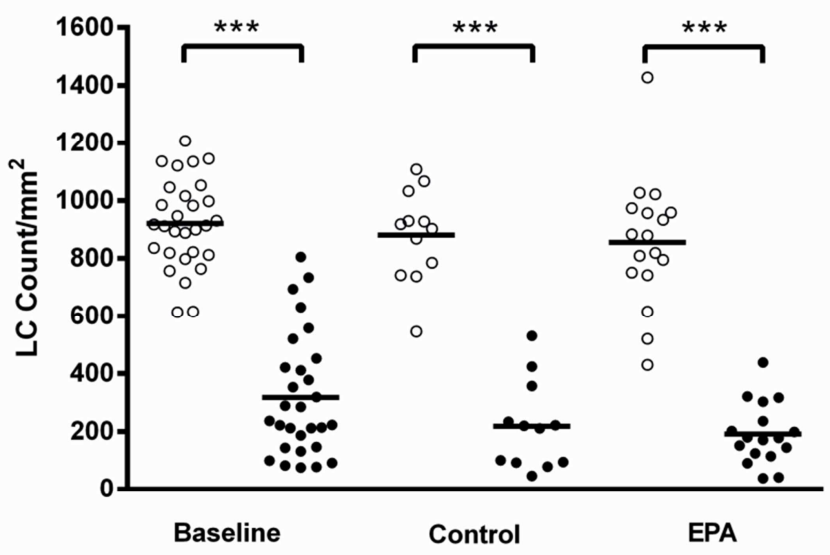

B No UVR UVR
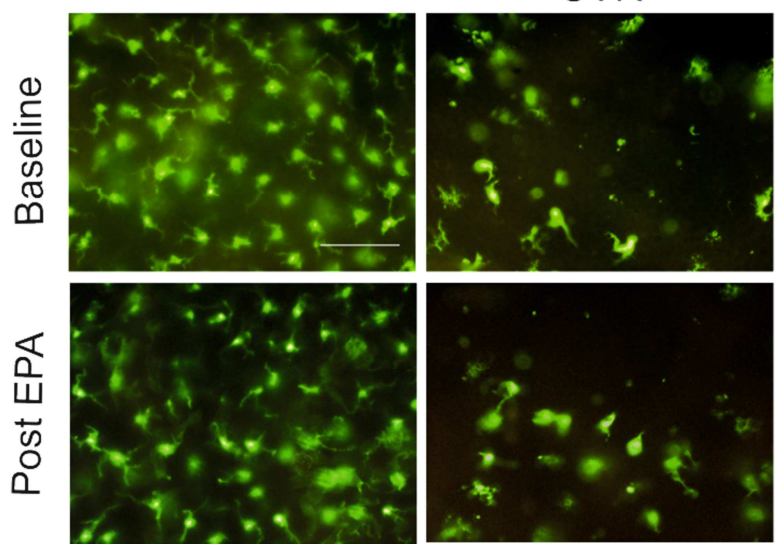
664

665

666
Fig 3.
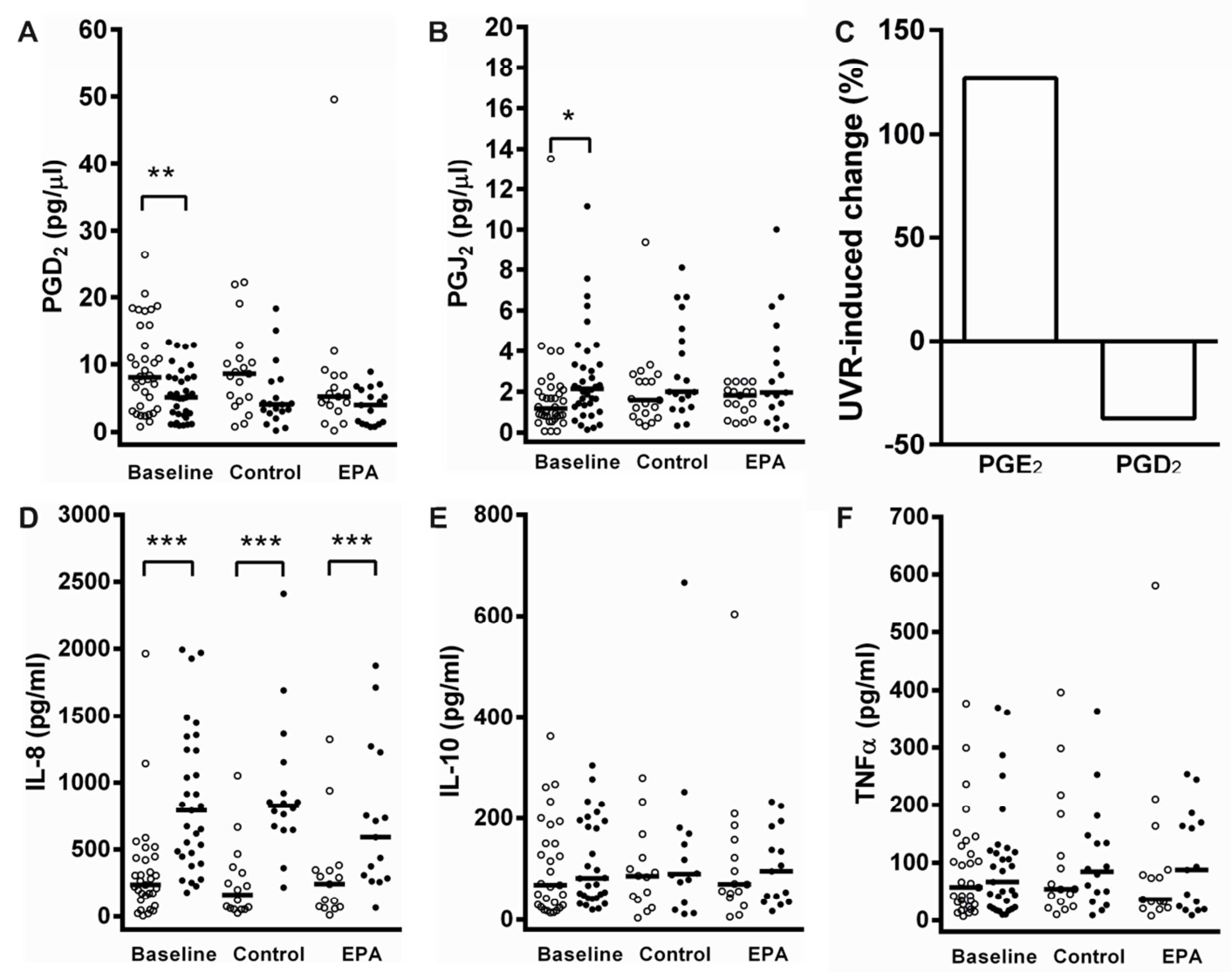